\title{
Data Dependence for Ishikawa Iteration When Dealing with Contractive-Like Operators
}

\author{
Ş. M. Şoltuz ${ }^{1,2}$ and Teodor Grosan ${ }^{3}$ \\ ${ }^{1}$ Departamento de Matematicas, Universidad de los Andes, Carrera 1 No. 18A-10, Bogota, Colombia \\ ${ }^{2}$ The Institute of Numerical Analysis, P.O. Box 68-1, Cluj-Napoca, Romania \\ ${ }^{3}$ Department of Applied Mathematics, Babes-Bolyai University, Cluj-Napoca, Romania
}

Correspondence should be addressed to Teodor Grosan, tgrosan@math.ubbcluj.ro

Received 13 February 2008; Accepted 27 May 2008

Recommended by Hichem Ben-El-Mechaiekh

We prove a convergence result and a data dependence for Ishikawa iteration when applied to contraction-like operators. An example is given, in which instead of computing the fixed point of an operator, we approximate the operator with a contractive-like one. For which it is possible to compute the fixed point, and therefore to approximate the fixed point of the initial operator.

Copyright (C) 2008 Ş. M. Şoltuz and T. Grosan. This is an open access article distributed under the Creative Commons Attribution License, which permits unrestricted use, distribution, and reproduction in any medium, provided the original work is properly cited.

\section{Introduction}

Let $X$ be a real Banach space; let $B \subset X$ be a nonempty convex closed and bounded set. Let $T, S: B \rightarrow B$ be two maps. For a given $x_{0}, u_{0} \in B$, we consider the Ishikawa iteration (see [1]) for $T$ and $S$ :

$$
\begin{array}{ll}
x_{n+1}=\left(1-\alpha_{n}\right) x_{n}+\alpha_{n} T y_{n}, & y_{n}=\left(1-\beta_{n}\right) x_{n}+\beta_{n} T x_{n}, \\
u_{n+1}=\left(1-\alpha_{n}\right) u_{n}+\alpha_{n} S v_{n}, & v_{n}=\left(1-\beta_{n}\right) u_{n}+\beta_{n} S u_{n},
\end{array}
$$

where $\left.\left\{\alpha_{n}\right\} \subset(0,1),\left\{\beta_{n}\right\} \subset 0,1\right)$, and

$$
\lim _{n \rightarrow \infty} \alpha_{n}=\lim _{n \rightarrow \infty} \beta_{n}=0, \sum_{n=1}^{\infty} \alpha_{n}=\infty \text {. }
$$

Set $\beta_{n}=0, \forall n \in N$, to obtain the Mann iteration, see [2].

The map $T$ is called Kannan mappings, see [3], if there exists $b \in(0,1 / 2)$ such that for all $x, y \in B$,

$$
\|T x-T y\| \leq b(\|x-T x\|+\|y-T y\|)
$$


Fixed Point Theory and Applications

Similar mappings are Chatterjea mappings, see [4], for which there exists $c \in(0,1 / 2)$ such that for all $x, y \in B$,

$$
\|T x-T y\| \leq c(\|x-T y\|+\|y-T x\|) .
$$

Zamfirescu collected these classes. He introduced the following definition, see [5].

Definition 1.1 (see $[5,6]$ ). The operator $T: X \rightarrow X$ satisfies condition $Z$ (Zamfirescu condition) if and only if there exist the real numbers $a, b, c$ satisfying $0<a<1,0<b, c<1 / 2$ such that for each pair $x, y$ in $X$, at least one condition is true:

(i) $\left(z_{1}\right)\|T x-T y\| \leq a\|x-y\|$,

(ii) $\left(z_{2}\right)\|T x-T y\| \leq b(\|x-T x\|+\|y-T y\|)$,

(iii) $\left(z_{3}\right)\|T x-T y\| \leq c(\|x-T y\|+\|y-T x\|)$.

It is known, see Rhoades [7], that $\left(z_{1}\right),\left(z_{2}\right)$, and $\left(z_{3}\right)$ are independent conditions. Consider $x, y \in B$. Since $T$ satisfies condition $Z$, at least one of the conditions from $\left(z_{1}\right),\left(z_{2}\right)$, and $\left(z_{3}\right)$ is satisfied. If $\left(z_{2}\right)$ holds, then

$$
\|T x-T y\| \leq b(\|x-T x\|+\|y-T y\|) \leq b(\|x-T x\|+(\|y-x\|+\|x-T x\|+\|T x-T y\|)) .
$$

Thus

$$
(1-b)\|T x-T y\| \leq b\|x-y\|+2 b\|x-T x\| .
$$

From $0 \leq b<1$ one obtains,

$$
\|T x-T y\| \leq \frac{b}{1-b}\|x-y\|+\frac{2 b}{1-b}\|x-T x\| .
$$

If $\left(z_{3}\right)$ holds, then one gets

$$
\|T x-T y\| \leq c(\|x-T y\|+\|y-T x\|) \leq c(\|x-T x\|+\|T x-T y\|+\|x-y\|+\|x-T x\|)
$$

Hence,

$$
(1-c)\|T x-T y\| \leq c\|x-y\|+2 c\|x-T x\|,
$$

that is,

$$
\|T x-T y\| \leq \frac{c}{1-c}\|x-y\|+\frac{2 c}{1-c}\|x-T x\|
$$

Denote

$$
\delta:=\max \left\{a, \frac{b}{1-b}, \frac{c}{1-c}\right\}
$$


to obtain

$$
0 \leq \delta<1
$$

Finally, we get

$$
\|T x-T y\| \leq \delta\|x-y\|+2 \delta\|x-T x\|, \quad \forall x, y \in B .
$$

Formula (1.14) was obtained as in [8].

Osilike and Udomene introduced in [9] a more general definition of a quasicontractive operator; they considered the operator for which there exists $L \geq 0$ and $q \in(0,1)$ such that

$$
\|T x-T y\| \leq q\|x-y\|+L\|x-T x\|, \quad \forall x, y \in B .
$$

Imoru and Olatinwo considered in [10], the following general definition. Because they failed to name them, we will call them here contractive-like operators.

Definition 1.2. One calls contractive-like the operator $T$ if there exist a constant $q \in 0,1$ ) and a strictly increasing and continuous function $\phi:[0, \infty) \rightarrow[0, \infty)$ with $\phi(0)=0$ such that for each $x, y \in X$,

$$
\|T x-T y\| \leq q\|x-y\|+\phi(\|x-T x\|) .
$$

In both papers $[9,10]$, the $T$-stability of Picard and Mann iterations was studied.

\section{Preliminaries}

The data dependence abounds in literature of fixed point theory when dealing with PicardBanach iteration, but is quasi-inexistent when dealing with Mann-Ishikawa iteration. As far as we know, the only data-dependence result concerning Mann-Ishikawa iteration is in [11]. There, the data dependence of Ishikawa iteration was proven when applied to contractions. In this note, we will prove data-dependence results for Ishikawa iteration when applied to the above contractive-like operators. Usually, Ishikawa iteration is more complicated but nevertheless more stable as Mann iteration. There is a classic example, see [12], in which Mann iteration does not converge while Ishikawa iteration does. This is the main reason for considering Ishikawa iteration in Theorem 3.2.

The following remark is obvious by using the inequality $(1-x) \leq \exp (x), \forall x \geq 0$.

Remark 2.1. Let $\left\{\theta_{n}\right\}$ be a nonnegative sequence such that $\theta_{n} \in(0,1], \forall n \in \mathbb{N}$. If $\sum_{n=1}^{\infty} \theta_{n}=\infty$, then $\prod_{n=1}^{\infty}\left(1-\theta_{n}\right)=0$.

The following is similar to lemma from [13]. (Note that another proof for this lemma [13] can be found in [11].)

Lemma 2.2. Let $\left\{a_{n}\right\}$ be a nonnegative sequence for which one supposes there exists $n_{0} \in \mathbb{N}$, such that for all $n \geq n_{0}$ one has satisfied the following inequality:

$$
a_{n+1} \leq\left(1-\lambda_{n}\right) a_{n}+\lambda_{n} \sigma_{n}
$$

where $\lambda_{n} \in(0,1), \forall n \in \mathbb{N}, \sum_{n=1}^{\infty} \lambda_{n}=\infty$, and $\sigma_{n} \geq 0 \forall n \in \mathbb{N}$. Then,

$$
0 \leq \lim _{n \rightarrow \infty} \sup a_{n} \leq \lim _{n \rightarrow \infty} \sup \sigma_{n} .
$$


Proof. There exists $n_{1} \in \mathbb{N}$ such that $\sigma_{n} \leq \lim \sup \sigma_{n}, \forall n \geq n_{1}$. Set $n_{2}=\max \left\{n_{0}, n_{1}\right\}$ such that the following inequality holds, for all $n \geq n_{2}$ :

$$
a_{n+1} \leq\left(1-\lambda_{n}\right)\left(1-\lambda_{n-1}\right) \cdots\left(1-\lambda_{n_{1}}\right) a_{n_{1}}+\lim _{n \rightarrow \infty} \sup \sigma_{n}
$$

Using the above Remark 2.1 with $\theta_{n}=\lambda_{n}$, we get the conclusion. In order to prove (2.3), consider (2.1) and the induction step:

$$
\begin{aligned}
a_{n+2} \leq & \left(1-\lambda_{n+1}\right) a_{n+1}+\lambda_{n+1} \sigma_{n+1} \leq\left(1-\lambda_{n+1}\right)\left(1-\lambda_{n}\right)\left(1-\lambda_{n-1}\right) \cdots\left(1-\lambda_{n_{1}}\right) a_{n_{1}} \\
& +\left(1-\lambda_{n+1}\right) \lim _{n \rightarrow \infty} \sup \sigma_{n}+\lambda_{n+1} \sigma_{n+1} \\
= & \left(1-\lambda_{n+1}\right)\left(1-\lambda_{n}\right)\left(1-\lambda_{n-1}\right) \cdots\left(1-\lambda_{n_{1}}\right) a_{n_{1}}+\lim _{n \rightarrow \infty} \sup \sigma_{n} .
\end{aligned}
$$

\section{Main results}

Theorem 3.1. Let $X$ be a real Banach space, $B \subset X$ a nonempty convex and closed set, and $T: B \rightarrow B a$ contractive-like map with $x^{*}$ being the fixed point. Then for all $x_{0} \in B$, the iteration (1.1) converges to the unique fixed point of $T$.

Proof. The uniqueness comes from (1.16); supposing we have two fixed points $x^{*}$ and $y^{*}$, we get

$$
\left\|x^{*}-y^{*}\right\|=\left\|T x^{*}-T y^{*}\right\| \leq q\left\|x^{*}-y^{*}\right\|+\phi\left(\left\|x^{*}-T x^{*}\right\|\right)=q\left\|x^{*}-y^{*}\right\|
$$

that is, $(1-q)\left\|x^{*}-y^{*}\right\|=0$. From (1.1) and (1.16) we obtain

$$
\begin{aligned}
\left\|x_{n+1}-x^{*}\right\| & \leq\left(1-\alpha_{n}\right)\left\|x_{n}-x^{*}\right\|+\alpha_{n}\left\|T y_{n}-T x^{*}\right\| \\
& \leq\left(1-\alpha_{n}\right)\left\|x_{n}-x^{*}\right\|+\alpha_{n} q\left\|y_{n}-x^{*}\right\| \\
& \leq\left(1-\alpha_{n}\right)\left\|x_{n}-x^{*}\right\|+\alpha_{n} q\left(1-\beta_{n}\right)\left\|x_{n}-x^{*}\right\|+q \alpha_{n} \beta_{n}\left\|T x_{n}-T x^{*}\right\| \\
& \left.\leq\left(1-\alpha_{n}(1-q)\right)\left(1-(1-q) \beta_{n}\right)\right)\left\|x_{n}-x^{*}\right\| \\
& \leq\left(1-\alpha_{n}(1-q)\right)\left\|x_{n}-x^{*}\right\| \leq \cdots \leq\left(\prod_{k=1}^{n}\left(1-\alpha_{k} q\right)\right)\left\|x_{0}-x^{*}\right\| .
\end{aligned}
$$

Use Remark 2.1 with $\theta_{k}=\alpha_{k} q$ to obtain the conclusion.

This result allows us to formulate the following data dependence theorem.

Theorem 3.2. Let $X$ be a real Banach space, let $B \subset X$ be a nonempty convex and closed set, and let $\varepsilon>0$ be a fixed number. If $T: B \rightarrow B$ is a contractive-like operator with the fixed point $x^{*}$ and $S: B \rightarrow B$ is an operator with a fixed point $u^{*}$, (supposed nearest to $\left.x^{*}\right)$, and if the following relation is satisfied:

$$
\|T z-S z\| \leq \varepsilon, \quad \forall z \in B
$$


then

$$
\left\|x^{*}-u^{*}\right\| \leq \frac{\varepsilon}{1-q}
$$

Proof. From (1.1) and (1.2), we have

$$
x_{n+1}-u_{n+1}=\left(1-\alpha_{n}\right)\left(x_{n}-u_{n}\right)+\alpha_{n}\left(T y_{n}-S v_{n}\right)
$$

Thus

$$
\begin{aligned}
\left\|x_{n+1}-u_{n+1}\right\|= & \left\|\left(1-\alpha_{n}\right)\left(x_{n}-u_{n}\right)+\alpha_{n}\left(S v_{n}-T y_{n}\right)\right\| \\
\leq & \left(1-\alpha_{n}\right)\left\|x_{n}-u_{n}\right\|+\alpha_{n}\left\|S v_{n}-T v_{n}+T v_{n}-T y_{n}\right\| \\
\leq & \left(1-\alpha_{n}\right)\left\|x_{n}-u_{n}\right\|+\alpha_{n}\left\|T v_{n}-S v_{n}\right\|+\alpha_{n}\left\|T v_{n}-T y_{n}\right\| \\
\leq & \left(1-\alpha_{n}\right)\left\|x_{n}-u_{n}\right\|+\alpha_{n} \varepsilon+q \alpha_{n}\left\|y_{n}-v_{n}\right\|+\alpha_{n} \phi\left(\left\|y_{n}-T y_{n}\right\|\right) \\
\leq & \left(1-\alpha_{n}\right)\left\|x_{n}-u_{n}\right\|+\alpha_{n} \varepsilon+q \alpha_{n}\left(1-\beta_{n}\right)\left\|x_{n}-u_{n}\right\|+q \alpha_{n} \beta_{n}\left\|T x_{n}-S u_{n}\right\|+\alpha_{n} \phi\left(\left\|y_{n}-T y_{n}\right\|\right) \\
\leq & \left(1-\alpha_{n}\right)\left\|x_{n}-u_{n}\right\|+\alpha_{n} \varepsilon+q \alpha_{n}\left(1-\beta_{n}\right)\left\|x_{n}-u_{n}\right\| \\
& +\alpha_{n} \beta_{n} q\left(\left\|T x_{n}-T u_{n}\right\|+\left\|T u_{n}-S u_{n}\right\|\right)+\alpha_{n} \phi\left(\left\|y_{n}-T y_{n}\right\|\right) \\
\leq & \left(1-\alpha_{n}\right)\left\|x_{n}-u_{n}\right\|+\alpha_{n} \varepsilon+q \alpha_{n}\left(1-\beta_{n}\right)\left\|x_{n}-u_{n}\right\| \\
& +q^{2} \alpha_{n} \beta_{n}\left\|x_{n}-u_{n}\right\|+q \alpha_{n} \beta_{n} \phi\left(\left\|x_{n}-T x_{n}\right\|\right)+q \alpha_{n} \beta_{n} \varepsilon+\alpha_{n} \phi\left(\left\|y_{n}-T y_{n}\right\|\right) \\
= & \left(1-\alpha_{n}\left(1-q\left(1-\beta_{n}\right)-\beta_{n} q^{2}\right)\right)\left\|x_{n}-u_{n}\right\|+\alpha_{n} \varepsilon+q \alpha_{n} \beta_{n} \varepsilon \\
& +q \alpha_{n} \beta_{n} \phi\left(\left\|x_{n}-T x_{n}\right\|\right)+\alpha_{n} \phi\left(\left\|y_{n}-T y_{n}\right\|\right) \\
= & \left(1-\alpha_{n}(1-q)\left(1+q \beta_{n}\right)\right)\left\|x_{n}-u_{n}\right\|+\alpha_{n}\left(q \beta_{n} \phi\left(\left\|x_{n}-T x_{n}\right\|\right)+\phi\left(\left\|y_{n}-T y_{n}\right\|\right)+q \beta_{n} \varepsilon+\varepsilon\right) \\
\leq & \left(1-\alpha_{n}(1-q)\right)\left\|x_{n}-u_{n}\right\|+\left(\alpha_{n}(1-q)\right) \frac{q \beta_{n} \phi\left(\left\|x_{n}-T x_{n}\right\|\right)+\phi\left(\left\|y_{n}-T y_{n}\right\|\right)+q \beta_{n} \varepsilon+\varepsilon}{1-q}
\end{aligned}
$$

Note that $\lim _{n \rightarrow \infty} \phi\left(\left\|x_{n}-T x_{n}\right\|\right)=\lim _{n \rightarrow \infty} \phi\left(\left\|y_{n}-T y_{n}\right\|\right)=0$ because $\phi$ is a continuous map and both $\left\{x_{n}\right\},\left\{y_{n}\right\}$ converge to the fixed point of $T$. Set

$$
\begin{aligned}
& \lambda_{n}:=\alpha_{n}(1-q), \\
& \sigma_{n}:=\frac{q \beta_{n} \phi\left(\left\|x_{n}-T x_{n}\right\|\right)+\phi\left(\left\|y_{n}-T y_{n}\right\|\right)+q \beta_{n} \varepsilon+\varepsilon}{1-q}
\end{aligned}
$$


and use Lemma 2.2 to obtain the conclusion

$$
\left\|x^{*}-u^{*}\right\| \leq \frac{\varepsilon}{1-q} \text {. }
$$

Remark 3.3. (i) Set $\beta_{n}=0, \forall n \in \mathbb{N}$, to obtain the data dependence for Mann iteration.

(ii) The Zamfirescu operators and implicitly (Chatterjea and Kannan) are contractive-like operators, therefore our Theorem 3.2 remains true for these classes.

\section{Numerical example}

The following example follows the example from [8].

Example 4.1. Let $T: \mathbb{R} \rightarrow \mathbb{R}$ be given by

$$
\begin{aligned}
T x & =0, \quad \text { if } x \in(-\infty, 2] \\
& =-0.5, \quad \text { if } x \in(2,+\infty) .
\end{aligned}
$$

Then $T$ is contractive-like operator with $q=0.2$ and $\phi=$ identity.

Note the unique fixed point is 0 . Consider now the map $S: R \rightarrow R$,

$$
\begin{aligned}
S x & =1, \quad \text { if } x \in(-\infty, 2] \\
& =-1.5, \quad \text { if } x \in(2,+\infty)
\end{aligned}
$$

with the unique fixed point 1 . Take $\varepsilon$ to be the distance between the two maps as follows:

$$
\|S x-T x\| \leq 1, \quad \forall x \in \mathbb{R} .
$$

Set $u_{0}=x_{0}=0, \alpha_{n}=\beta_{n}=1 /(n+1)$. Independently of above theory, the Ishikawa iteration applied to $S$, leads to

$\begin{array}{cc}\text { Iteration step } & \text { Ishikawa iteration } \\ 1 & 0.5 \\ 10 & 0.9 \\ 100 & 0.99\end{array}$

Note that for $n=1$,

$$
0.5=\frac{1}{n+1} 0+\frac{1}{n+1} S\left(\frac{1}{2}\right)
$$

since $y_{1}=(1 /(n+1)) 0+(1 / n+1) 1=1 / 2$. (The above computations can be obtained also by using a Matlab program.) This leads us to "conclude" that Ishikawa iteration applied to $S$ converges to fixed point, $\left(x^{*}=1\right)$. Eventually, one can see that the distance between the two fixed points is one. Actually, without knowing the fixed point of $S$ (and without computing it), via Theorem 3.2, we can do the following estimate for it:

$$
\left\|x^{*}-u^{*}\right\| \leq \frac{1}{1-q}=\frac{1}{1-0.2}=\frac{10}{8}=1.2
$$

As a conclusion, instead of computing fixed points of $S$, choose $T$ more closely to $S$ and the distance between the fixed points will shrink too. 


\section{Acknowledgments}

The authors are indebted to referee for carefully reading the paper and for making useful suggestions. This work was supported by CEEX ET 90/2006-2008.

\section{References}

[1] S. Ishikawa, "Fixed points by a new iteration method," Proceedings of the American Mathematical Society, vol. 44, no. 1, pp. 147-150, 1974.

[2] W. R. Mann, "Mean value methods in iteration," Proceedings of the American Mathematical Society, vol. 4, no. 3, pp. 506-510, 1953.

[3] R. Kannan, "Some results on fixed points," Bulletin of the Calcutta Mathematical Society, vol. 60, pp. 71-76, 1968.

[4] S. K. Chatterjea, "Fixed-point theorems," Comptes Rendus de l'Académie Bulgare des Sciences, vol. 25, pp. 727-730, 1972.

[5] T. Zamfirescu, "Fix point theorems in metric spaces," Archiv der Mathematik, vol. 23, no. 1, pp. 292-298, 1972.

[6] B. E. Rhoades, "Fixed point iterations using infinite matrices," Transactions of the American Mathematical Society, vol. 196, pp. 161-176, 1974.

[7] B. E. Rhoades, "A comparison of various definitions of contractive mappings," Transactions of the American Mathematical Society, vol. 226, pp. 257-290, 1977.

[8] V. Berinde, "On the convergence of the Ishikawa iteration in the class of quasi contractive operators," Acta Mathematica Universitatis Comenianae, vol. 73, no. 1, pp. 119-126, 2004.

[9] M. O. Osilike and A. Udomene, "Short proofs of stability results for fixed point iteration procedures for a class of contractive-type mappings," Indian Journal of Pure and Applied Mathematics, vol. 30, no. 12, pp. 1229-1234, 1999.

[10] C. O. Imoru and M. O. Olatinwo, "On the stability of Picard and Mann iteration processes," Carpathian Journal of Mathematics, vol. 19, no. 2, pp. 155-160, 2003.

[11] S.. M. Şoltuz, "Data dependence for Ishikawa iteration," Lecturas Matemáticas, vol. 25, no. 2, pp. 149$155,2004$.

[12] C. E. Chidume and S. A. Mutangadura, "An example of the Mann iteration method for Lipschitz pseudocontractions," Proceedings of the American Mathematical Society, vol. 129, no. 8, pp. 2359-2363, 2001.

[13] J. A. Park, "Mann-iteration process for the fixed point of strictly pseudocontractive mapping in some Banach spaces," Journal of the Korean Mathematical Society, vol. 31, no. 3, pp. 333-337, 1994. 